\title{
Influence of Atmospheric Stability on Wind-Turbine Wakes: A Large-Eddy Simulation Study
}

\author{
Mahdi Abkar ${ }^{1, a)}$ and Fernando Porté-Agel $\left.{ }^{1, b}\right)$ \\ École Polytechnique Fédérale de Lausanne (EPFL), Wind Engineering \\ and Renewable Energy Laboratory (WiRE), EPFL-ENAC-IIE-WIRE, \\ CH-1015 Lausanne
}

In this study, large-eddy simulation is combined with a turbine model to investigate the influence of atmospheric thermal stability on wind-turbine wakes. The simulation results show that atmospheric stability has a significant effect on the spatial distribution of the mean velocity deficit and turbulence statistics in the wake region as well as the wake meandering characteristics downwind of the turbine. In particular, the enhanced turbulence level associated with positive buoyancy under the convective condition leads to a relatively larger flow entrainment and, thus, a faster wake recovery. For the particular cases considered in this study, the growth rate of the wake is about 2.4 times larger for the convective case than for the stable one. Consistent with this result, for a given distance downwind of the turbine, wake meandering is also stronger under the convective condition compared with the neutral and stable cases. It is also shown that, for all the stability cases, the growth rate of the wake and wake meandering in the vertical direction are smaller compared with the ones in the lateral direction. This is mainly related to the different turbulence levels of the incoming wind in the different directions, together with the anisotropy imposed by the presence of the ground. It is also found that the wake velocity deficit is well characterized by a modified version of a recently proposed analytical model that is based on mass and momentum conservation and the assumption of a self-similar Gaussian distribution of the velocity deficit. Specifically, using a two-dimensional elliptical (instead of axisymmetric) Gaussian distribution allows to account for the different lateral and vertical growth rates, particularly in the convective case where the non-axisymmetry of the wake is stronger. Detailed analysis of the resolved turbulent kinetic energy budget in the wake reveals also that thermal stratification considerably affects the magnitude and spatial distribution of the turbulence production, dissipation and transport terms.

\section{INTRODUCTION}

A wind turbine extracts energy from the incoming wind, which results in a region behind the turbine with a strong wind velocity deficit and an enhanced turbulence level with respect to the upstream flow - a wake. The velocity deficit in the wake decreases with distance, as faster moving air is entrained gradually into the wake ${ }^{1,2}$.

Within a wind farm, the wind speed in the turbine wakes does not recover to its upstream unperturbed value and, thus, the waked turbines produce less energy. For instance, in a study of power output data from two large offshore wind farms with different streamwise spacings, 10.5 and 7 rotor diameters, Barthelmie et al. ${ }^{3}$ reported power reduction up to $40 \%$ for the waked turbines with respect to the unwaked ones. In addition to a significant decrease of the power production, the waked turbines experience an increase in fatigue loads related to the high turbulence level in the wakes. Therefore, detailed understanding and accurate prediction of wind-turbine wakes and their effects in wind farms are needed for optimizing the design of wind farms, specifically for maximizing the energy production and minimizing the maintenance costs due to fatigue loads.

\footnotetext{
a) Electronic mail: mahdi.abkar@epfl.ch

b) Electronic mail: fernando.porte-agel@epfl.ch
} 
Wind turbines operate in the lowest region of the atmospheric boundary layer (ABL). As a result, the structure and evolution of wind-turbine wakes are affected by the characteristics of the ABL flow such as the wind speed, wind shear and turbulence levels. In turn, these ABL characteristics, and thus the turbine wakes, are affected by land/sea surface characteristics, such as the aerodynamic roughness and topography, as well as thermal stratification. According to the thermal stratification and the dominant mechanisms of turbulence generation, the atmospheric boundary layer can be classified into three types: neutral, convective and stable. In the neutral boundary layer (NBL), there is little heating or cooling at the surface. Hence, the mean potential temperature is approximately constant with height and turbulence is mainly produced by shear in proximity to the surface. The neutrally-stratified ABL is observed during relatively short transition periods after sunset, or in windy conditions with a complete cloud cover. The unstable or convective boundary layer (CBL) is typically observed during day time, when the surface is warmer than the air. Under unstable conditions, heat transfer between the surface and the air produces positive buoyancy, which enhances the turbulent kinetic energy (TKE) and the vertical transport of momentum, heat and moisture ${ }^{4}$. Finally, the stably-stratified ABL occurs usually at night, in response to surface cooling by longwave radiation to outer space ${ }^{5}$. Under this atmospheric condition, turbulence is generated by shear and destroyed by negative buoyancy and viscosity. As a result, in the stable boundary layer (SBL), turbulence is weaker than in the neutral and convective cases.

Evidence of significant thermal stability effects on wind-turbine wake structure and power harvesting has been reported in full-scale field measurements as well as wind-tunnel tests of down-scaled wind-turbine models. Baker and Walker ${ }^{6}$ carried out measurements behind a 2.5-MW wind turbine at Goodnoe Hills, Washington. Their results showed a slower wake recovery under stable nighttime conditions characterized by relatively lower turbulence levels. Later, Magnusson and Smedman ${ }^{7}$ investigated the structure of the wakes downwind of a wind turbine in the Alsvik wind farm during different atmospheric stability conditions. They showed that the velocity deficit is a function of atmospheric stratification, with larger values in the stable conditions. Barthelmie and Jensen ${ }^{8}$ estimated wind-farm efficiency reduction up to $9 \%$ in the stable conditions compared with the unstable ones for the wind-speed range of 9-10 m/s at the Nysted wind farm in the Danish Baltic Sea. Iungo and Porté-Agel ${ }^{9}$ performed wind velocity measurements of the wake downwind of a 2 MW Enercon E-70 wind turbine with three scanning Doppler wind LiDARs under different atmospheric conditions. They showed that the wakes recover faster under the convective conditions compared with the neutral cases. Recently, Zhang et al. ${ }^{10}$ carried out wind-tunnel experiments of the down-scaled wind-turbine models to investigate the effect of thermal stability on wind-turbine wakes under the convective and neutral conditions. They found a smaller velocity deficit (around 15\% at the wake center) in the CBL compared with the wake of the same wind turbine in the neutral boundary layer. Hancock et al. ${ }^{11}$ also performed wind-tunnel measurements in the wake of a model wind turbine in neutral and stable boundary layers. They showed that under the stable condition, characterized by a lower turbulence level, the velocity deficit decreased more slowly compared to the neutral case.

Besides field measurements and wind-tunnel experiments of wind-turbine wakes, some numerical studies have also addressed the effect of atmospheric stability on the evolution of wind-turbine wakes and the wind-turbine performance. Keck et al. ${ }^{12}$ studied the effect of atmospheric stability on wake meandering and the power production by the turbines using a dynamic wake meandering (DWM) model. By comparing the DWM model results with the field data of the North Hoyle and OWEZ wind farms, they showed the wake losses were $10 \%$ smaller under very unstable conditions compared with neutral ones. In contrast, very stable stratification led to wake-induced power losses of about $12 \%$ higher than those under neutral conditions. Churchfield et al. ${ }^{13}$ performed numerical simulations to study the effect of convective atmospheric turbulence on wind turbine performance. In the simulations, they placed a second wind turbine 7 rotor diameters downwind of the first one and showed that the ratio of the power produced by the second turbine downwind relative to that 
of the upwind turbine is 15-20\% higher under the unstable condition compared with the neutral one. As illustrated above, atmospheric thermal stability cannot be viewed as a small perturbation to a basic neutral state. Thus, a detailed comprehension of the variability of wind turbine wakes under different ABL thermal regimes is crucial.

The present work aims to investigate systematically the effect of ABL thermal stability on wind-turbine wake characteristics using a recently-developed large-eddy simulation (LES) framework. In this framework, the Lagrangian scale-dependent dynamic model ${ }^{14}$ is used to parameterize the subgrid-scale (SGS) fluxes and the actuator-disk model with rotation is applied to calculate the turbine-induced lift and drag forces ${ }^{15,16}$. Numerical experiments are carried out under different atmospheric stability conditions in order to examine the effect of thermal stratification on wind-turbine wakes. A short introduction of the LES framework and the numerical setup is presented in Section II. The spatial distribution of the mean velocity, turbulence intensity, and turbulent momentum fluxes is shown and discussed in Section III A. In Section III B, our simulation results are employed to develop a new analytical model, based on a modification of the recently proposed model of Bastankhah and Porté-Agel ${ }^{17}$, to predict the spatial distribution of the velocity deficit downwind of the turbine. Detailed analysis of the resolved-scale turbulent kinetic energy (TKE) budget is presented in Section III C and, finally, a summary and conclusions are provided in Section IV.

\section{LARGE-EDDY SIMULATION FRAMEWORK}

\section{A. LES Governing Equations}

LES solves the filtered continuity equation, the filtered incompressible Navier-Stokes equations, and the filtered transport equation for potential temperature:

$$
\begin{gathered}
\frac{\partial \widetilde{u}_{i}}{\partial x_{i}}=0 \\
\frac{\partial \widetilde{u}_{i}}{\partial t}+\widetilde{u}_{j} \frac{\partial \widetilde{u}_{i}}{\partial x_{j}}=-\frac{\partial \tilde{p}^{*}}{\partial x_{i}}-\frac{\partial \tau_{i j}^{d}}{\partial x_{j}}+\delta_{i 3} g \frac{\widetilde{\theta}-\langle\widetilde{\theta}\rangle}{\theta_{0}}-\frac{f_{i}}{\rho}+F_{p} \delta_{i 1} \\
\frac{\partial \widetilde{\theta}}{\partial t}+\widetilde{u}_{j} \frac{\partial \widetilde{\theta}}{\partial x_{j}}=-\frac{\partial q_{j}}{\partial x_{j}}
\end{gathered}
$$

where the tilde represents a spatial filtering at scale $\widetilde{\Delta}, t$ is time, $\widetilde{u}_{i}$ is the instantaneous resolved velocity in the $i$-direction (with $i=1,2,3$ corresponding to the streamwise $(x)$, spanwise $(y)$ and vertical $(z)$ directions, respectively), $\widetilde{\theta}$ denotes the resolved potential temperature, $\theta_{0}$ is the reference temperature. The angle brackets represent a horizontal average, $g$ refers to the gravitational acceleration, $\delta_{i j}$ is the Kronecker delta, $\tilde{p}^{*}=\tilde{p} / \rho+\frac{1}{3} \tau_{k k}$ is the modified kinematic pressure. $f_{i}$ is a body force (per unit volume) used to model the effect of the turbine on the flow, $\rho$ is the fluid density, and $F_{p}$ is an imposed pressure gradient. $q_{j}$ denotes the SGS heat flux, $\tau_{i j}$ represents the kinematic SGS stress, and $\tau_{i j}^{d}$ is its deviatoric (trace-free) part. It should be noted that in the momentum conservation equation above, the buoyancy effects are accounted for via the Boussinesq approximation (see Stull ${ }^{4}$ pp. 81-85).

In LES, all turbulent structures larger than the filter scale $(\widetilde{\Delta})$ are resolved and the contribution of the unresolved small-scale eddies on the resolved field is parameterized using a SGS model. A common parameterization strategy in LES consists of computing the deviatoric part of the SGS stress with an eddy-viscosity model $^{18}$ :

$$
\tau_{i j}^{d}=\tau_{i j}-\frac{1}{3} \delta_{i j} \tau_{k k}=-2 \widetilde{\Delta}^{2} C_{S}^{2}|\widetilde{S}| \widetilde{S}_{i j}
$$


and the SGS heat flux with an eddy-diffusivity model:

$$
q_{j}=-\widetilde{\Delta}^{2} C_{S}^{2} \operatorname{Pr}_{s g s}^{-1}|\widetilde{S}| \frac{\partial \widetilde{\theta}}{\partial x_{j}}
$$

where $\tilde{S}_{i j}=\left(\partial \tilde{u}_{i} / \partial x_{j}+\partial \tilde{u}_{j} / \partial x_{i}\right) / 2$ is the resolved strain-rate tensor whose magnitude is $|\widetilde{S}|$, $\widetilde{\Delta}$ is the filter width, $C_{S}$ is the Smagorinsky coefficient, $C_{S}^{2} P r_{s g s}^{-1}$ is the lumped coefficient, and $P r_{s g s}$ is the SGS Prandtl number. Here, we employ the scale-dependent Lagrangian dynamic models ${ }^{14}$ to compute the local optimized value of the model coefficients without any ad hoc tuning. In contrast with the traditional dynamic models ${ }^{19,20}$, the scale-dependent dynamic models compute dynamically not only the value of the model coefficients in the eddy-viscosity and eddy-diffusivity models, but also the dependence of these coefficients with scale. More details on the formulation of scale-dependent dynamic models for the SGS fluxes can be found in Porté-Agel et al. ${ }^{21}$, Porté-Agel ${ }^{22}$, and Stoll and Porté-Agel ${ }^{14}$.

\section{B. Wind-Turbine Parameterization}

To parameterize the turbine-induced forces, the actuator-disk model with rotation ${ }^{15}$ is used. This model is based on blade-element theory and, therefore, it does not require resolving the boundary-layer flow around the turbine-blade surfaces, which greatly reduces the computational cost requirements compared with full-scale blade-resolving CFD models. This theory considers that each blade of a wind turbine can be divided into $N$ blade elements which are assumed to behave aerodynamically as two-dimensional airfoils and to have no radial action on the flow. Through this model, both lift and drag forces acting on each blade element (see Figure 1) are parameterized based on the relative wind velocity, the geometry of a blade airfoil, and tabulated airfoil data. Unlike the standard actuator-disk model, which assumes the loads are distributed uniformly over the rotor disk and acting only in the axial direction, the actuator-disk model with rotation includes the effect of turbine-induced flow rotation as well as the non-uniform force distribution.

To determine the forces acting on the rotor disk, we consider an element with a differential size of $d A=r d \theta d r$ on a cylindrical mesh system. The resulting force per unit rotor area is given by:

$$
\mathbf{f}_{d i s k}=\frac{d \mathbf{F}}{d A}=\frac{1}{2} \rho V_{r e l}^{2} \frac{B c}{2 \pi r}\left(C_{L} \mathbf{e}_{L}+C_{D} \mathbf{e}_{D}\right),
$$

where $B$ is the number of blades, $c$ is the local chord length, $C_{L}$ and $C_{D}$ are the lift and drag coefficients, respectively, $r$ is the radial distance between the hub and the center of the blade element, and $\mathbf{e}_{L}$ and $\mathbf{e}_{D}$ denote the unit vectors in the directions of the lift and the drag, respectively. $V_{r e l}$ is the magnitude of the local velocity relative to the rotating blade and defined as $\mathbf{V}_{\text {rel }}=\left(V_{x}, \Omega r-V_{\theta}\right)$, where $V_{x}=V_{x}(r, \theta)$ and $V_{\theta}=V_{\theta}(r, \theta)$ are axial and tangential velocities of the incident flow at the blades, respectively, in the inertial frame of reference, and $\Omega$ is the turbine angular velocity. In Figure 1, $\alpha$ is the angle of attack, defined as $\alpha=\varphi-\gamma$, where $\varphi=\tan ^{-1}\left(V_{x} /\left(\Omega r-V_{\theta}\right)\right)$ is the angle between $\mathbf{V}_{r e l}$ and the rotor plane and $\gamma$ is the local pitch angle. In LESs of flow through wind turbines, the tangential $\left(V_{\theta}\right)$ and the axial $\left(V_{x}\right)$ velocities at the rotor plane are readily available during the simulation. Hence, the local velocity relative to the rotating blade $\left(\mathbf{V}_{r e l}\right)$ and the angle of attack $\alpha=\varphi-\gamma$ can be computed. Then, the resulting force is obtained using Equation 6. It should be mentioned that in the model based on blade-element theory, the lift and drag coefficients are obtained from the tabulated airfoil data. As a result, the performance of this method is sensitive to the input blade section aerodynamic data. Wu and Porté-Agel ${ }^{23}$ provided details of the Vestas V80-2MW wind turbine, which is used in this study, in a simulation of an operational wind farm. 


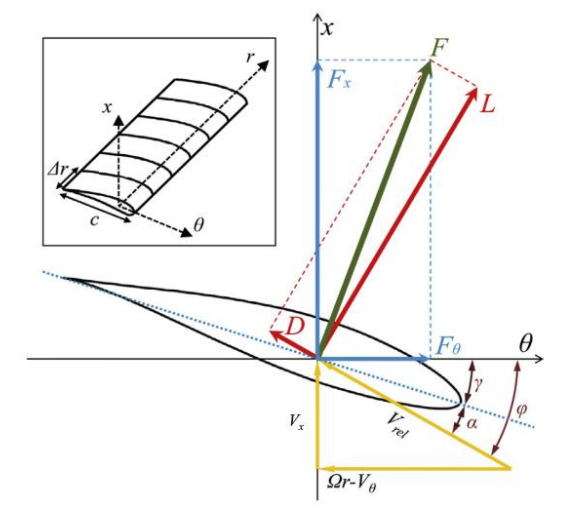

FIG. 1. A cross-sectional airfoil element.

\section{Numerical Setup}

The LES code used in this study is a modified version of the one described by Albertson and Parlange ${ }^{24}$, Porté-Agel et al. ${ }^{21}$, Stoll and Porté-Agel ${ }^{14}$, Porté-Agel et al. ${ }^{16}$, Wu and Porté-Agel ${ }^{15}$ and Porté-Agel et al. ${ }^{25}$. In the simulations, tuning-free Lagrangian scaledependent dynamic models are used to model the subgrid-scale turbulent fluxes, and the turbine-induced forces are parameterized using the above-mentioned actuator-disk model. Since the Reynolds number of the ABL is very high, near-ground viscous processes are not resolved, and the viscous term is neglected in the momentum equation.

The domain is divided uniformly into $N_{x} \times N_{y} \times N_{z}$ grid points with a spatial resolution of $\Delta x \times \Delta y \times \Delta z$ in the streamwise, spanwise and wall-normal directions, respectively. The grid planes are staggered in the vertical direction, with the first vertical velocity plane at a distance $\Delta_{z}$ from the surface, and the first horizontal velocity plane $\Delta_{z} / 2$ from the surface. The filter width is computed using the common formulation $\widetilde{\Delta}=\left(\Delta_{x} \Delta_{y} \Delta_{z}\right)^{1 / 3}$. The horizontal domain spans, $L_{x}=3200 \mathrm{~m}$ and $L_{y}=1600 \mathrm{~m}$, are parallel and perpendicular to the mean wind direction, respectively. The height of the computational domain is set to $L_{z}=1000 \mathrm{~m}$. A constant streamwise pressure gradient is used to drive the flow within the boundary layer, which has a height of $\delta$. In the present work, we consider different values for the boundary-layer height $(\delta)$ under different stability conditions. This is due to the fact that the boundary layer is typically deeper under convective conditions and is shallower under stable ones ${ }^{26}$. The chosen values for the height of the boundary layer are consistent with the boundary-layer height observed by Peña et al. ${ }^{27}$ at the National Test for Wind Turbines at Hovsore, Denmark.

The vertical derivatives are approximated with second-order central differences and the horizontal directions are discretized pseudo-spectrally, which requires periodic boundary conditions. To avoid the downwind flow affecting the flow upwind of the wind turbine due to the periodic boundary conditions, a buffer zone upstream the turbine is employed to adjust the flow from the downwind condition to that of a fully turbulent boundarylayer inflow condition. The inflow condition is obtained from separate (without turbine) simulations of fully-developed ABL flows over horizontally-homogeneous flat surfaces ${ }^{15,28,29}$. Full dealiasing of the nonlinear terms is obtained by padding and truncation according to the $3 / 2$ rule $^{30}$. The time advancement is carried out using a second-order-accurate AdamsBashforth scheme ${ }^{31}$.

The finite-difference scheme in the vertical direction requires specification of boundary conditions at the top and bottom of the domain. The upper boundary condition is a stress/flux-free condition. At the bottom surface, the instantaneous wall stress is related to the velocity at the first vertical node through the application of the Monin-Obukhov 
similarity theory ${ }^{32}$ as follows ${ }^{33,34}$ :

$$
\left.\tau_{i 3}\right|_{w}=-u_{*}^{2} \frac{\tilde{u}_{i}}{\tilde{u}_{r}}=-\left(\frac{\tilde{u}_{r} \kappa}{\ln \left(z / z_{o}\right)-\Psi_{M}}\right)^{2} \frac{\tilde{u}_{i}}{\tilde{u}_{r}}
$$

where $\left.\tau_{i 3}\right|_{w}$ is the instantaneous local wall stress, $u_{*}$ is the friction velocity, $z_{o}=0.05 \mathrm{~m}$ is the aerodynamic surface roughness, $\kappa$ is the von Kármán constant, and $\tilde{u}_{r}$ is the local filtered horizontal velocity at the first level $z=\Delta z / 2$. In a similar manner, the surface heat flux is computed as:

$$
\left.q_{3}\right|_{w}=\frac{u_{*} \kappa\left(\theta_{s}-\tilde{\theta}\right)}{\ln \left(z / z_{o}\right)-\Psi_{H}}
$$

where $\theta_{s}$ is the surface (ground level) potential temperature. $\Psi_{M}$ and $\Psi_{H}$ are the stability corrections for momentum and heat, respectively, and are defined as follows ${ }^{4,35}$ :

$$
\Psi_{M}= \begin{cases}-4.7 \frac{z}{L} & \text { for stable conditions } \\ 2 \ln \left[\frac{1}{2}(1+x)\right]+\ln \left[\frac{1}{2}\left(1+x^{2}\right)\right]-2 \tan ^{-1}[x]+\frac{\pi}{2} & \text { for unstable conditions }\end{cases}
$$

and

$$
\Psi_{H}= \begin{cases}-7.8 \frac{z}{L} & \text { for stable conditions, } \\ 2 \ln \left[\frac{1}{2}\left(1+x^{2}\right)\right] & \text { for unstable conditions, }\end{cases}
$$

where $L=-\left(u_{*}^{3} \theta_{0}\right) /\left(\left.\kappa g q_{3}\right|_{w}\right)$ is the local Obukhov length, and $x=(1-15 z / L)^{1 / 4}$. In the present work, we directly specify the values of the imposed pressure gradient $\left(F_{p}=u_{*}^{2} / \delta\right)$ and the surface heat flux $\left(\left.q_{3}\right|_{w}\right)$ such that the incoming flows have the same mean velocity at the hub height equal to $U_{h u b} \approx 8 \mathrm{~ms}^{-1}$ but different stability conditions. In the simulations, Vestas V80-2MW wind turbines, with a rotor diameter $(D)$ of $80 \mathrm{~m}$ and a hub-height $\left(z_{h}\right)$ of $70 \mathrm{~m}$, are immersed in the flow. Based on the operational records of Vestas V80-2MW wind turbines reported by Hansen et al. ${ }^{36}$, the turbines reach a mean angular velocity of $16.1 \mathrm{rpm}$ when the incoming (upwind) mean wind speed at hub height is $U_{h u b} \approx 8 \mathrm{~ms}^{-1}$. The code is run for a long-enough time to guarantee that quasi-steady conditions are reached. It should be mentioned that two different spatial resolutions are used to test the resolution sensitivity of the simulation results. The key parameters of the various LES cases are summarized in Table. I.

TABLE I. Key parameters of the various LES cases.

\begin{tabular}{lccccccc}
\hline \hline ABL & Stability & $L$ & $u_{*}$ & $\left.q_{3}\right|_{w}$ & $\delta$ & $\Delta_{x} \times \Delta_{y} \times \Delta_{z}$ & $\Delta_{x} \times \Delta_{y} \times \Delta_{z}$ \\
cases & condition & $(m)$ & $\left(\mathrm{ms}^{-1}\right)$ & $\left(\mathrm{Kms}^{-1}\right)$ & $(\mathrm{m})$ & $\left(\mathrm{m}^{3}\right) \mathrm{GR} 1$ & $\left(\mathrm{~m}^{3}\right) \mathrm{GR} \mathrm{2}$ \\
\hline CBL & Convective & -150 & 0.46 & 0.048 & 750 & $16 \times 10 \times 5$ & $25.6 \times 16 \times 8$ \\
NBL & Neutral & $\infty$ & 0.4 & 0 & 500 & $16 \times 10 \times 5$ & $25.6 \times 16 \times 8$ \\
SBL & Stable & 150 & 0.31 & -0.015 & 250 & $16 \times 10 \times 5$ & $25.6 \times 16 \times 8$ \\
\hline \hline
\end{tabular}

\section{RESULTS}

\section{A. Mean velocity, turbulence intensity and turbulent momentum fluxes}

In this section, we present results from the large-eddy simulations of the wake flows behind the wind turbine under different stability conditions. These results are obtained from the simulations using the finer grid resolution (i.e, GR 1 in Table I). In order to characterize the structure of the turbulent wake flow, the spatial distribution of key turbulence statistics 
such as time-averaged streamwise velocity $U\left(\mathrm{~ms}^{-1}\right)$, normalized velocity deficit $\Delta U / U_{h u b}$, turbulence intensity, and turbulent momentum fluxes are shown. In all the cases, the incoming wind has the same wind speed $U_{h u b} \approx 8 \mathrm{~ms}^{-1}$ at the hub height but different mean wind shears and turbulence intensity levels. The main characteristics of the simulated ABLs under different stability conditions are shown in Figure 2. As expected, under the stable condition, the mean wind shear is stronger compared with the neutral and convective cases (Figure 2a). In addition, the negative surface buoyancy fluxes in the stable condition damps the turbulence, which leads to lower turbulence levels $\left(I_{u_{i}}=\sigma_{u_{i}} / U_{h u b}\right)$ in all three directions (Figure 2d-f). Similarly, the kinematic shear stress $-\overline{u^{\prime} w^{\prime}}$ in the stable condition is lower compared with the neutral and unstable ones (Figure 2c). These simulated velocity and temperature fields are then used as inflows to the wind-turbine wake simulations.
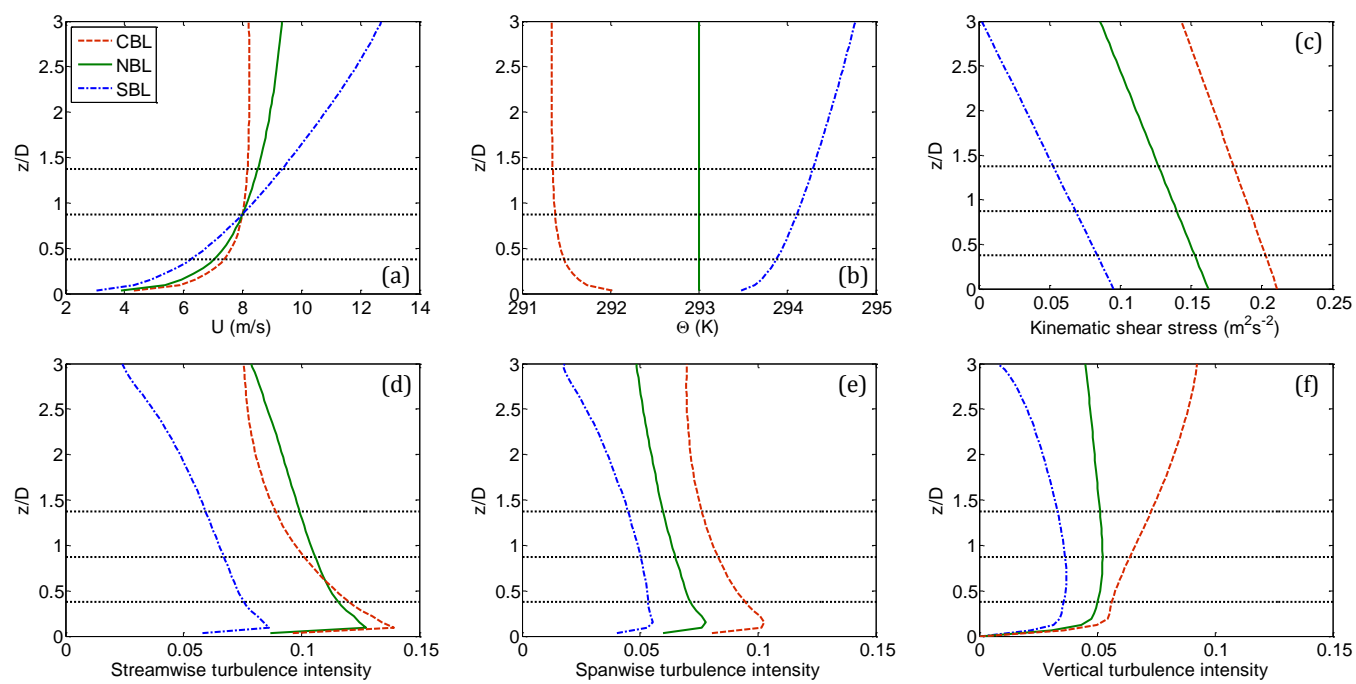

FIG. 2. Vertical profiles of a) the time-averaged streamwise velocity $U\left(\mathrm{~ms}^{-1}\right)$, b) the time-averaged potential temperature $\Theta(K)$, c) the kinematic shear stress $-\overline{u^{\prime} w^{\prime}}\left(m^{2} s^{-2}\right)$, d) the streamwise turbulence intensity $I_{u}=\sigma_{u} / U_{h u b}$, e) the spanwise turbulence intensity $I_{v}=\sigma_{v} / U_{h u b}$, f) the vertical turbulence intensity $I_{w}=\sigma_{w} / U_{h u b}$ of the incoming ABL flows. The horizontal dotted lines show the top-tip, hub, and bottom-tip heights.

Figures 3 and 4 display the time-averaged streamwise velocity contours in a vertical $x-z$ plane through the center of the turbines as well as in a horizontal $x-y$ plane at the turbine hub height, respectively. The simulation results indicate that atmospheric stability significantly affects the mean velocity distribution in the turbine wakes. In particular, in the convective boundary layer, with a higher turbulence level, the wake recovers faster. This is due to the fact that, in the unstable condition, the positive buoyancy flux at the surface creates thermal instabilities which enhance the turbulent kinetic energy (TKE) and the turbulent mixing. In contrast, in the stable condition, the negative buoyancy flux at the surface damps the turbulence and, consequently, reduces the turbulent mixing. As a result, it takes a longer distance for the wake to recover (i.e., larger wake region). It is important to note that, although the streamwise turbulence intensity $I_{u}$ is lower under the convective condition compared with the neutral one (Figure 2d), the wake recovery is faster under the convective condition. It should be mentioned that it is a common practice to use the streamwise turbulence intensity to describe single-wake behaviour including the velocity deficit and the width of the wake ${ }^{37,38}$. These results indicate that the streamwise turbulence intensity alone is not sufficient to describe the impact of atmospheric stability on wind-turbine wakes, and the turbulence intensities in all three directions must be considered.

The spanwise and vertical profiles of the velocity deficit $\Delta U / U_{h u b}$ through the center of the wakes are shown in Figure 5, where $\Delta U=U_{\text {inflow }}-U$ is the time-averaged velocity deficit, 


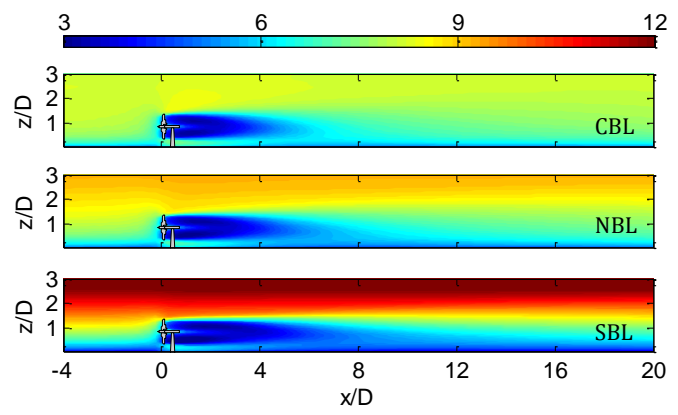

FIG. 3. Contours of the time-averaged streamwise velocity $U\left(m s^{-1}\right)$ in the middle vertical $x-z$ plane perpendicular to the turbines for different stability conditions.

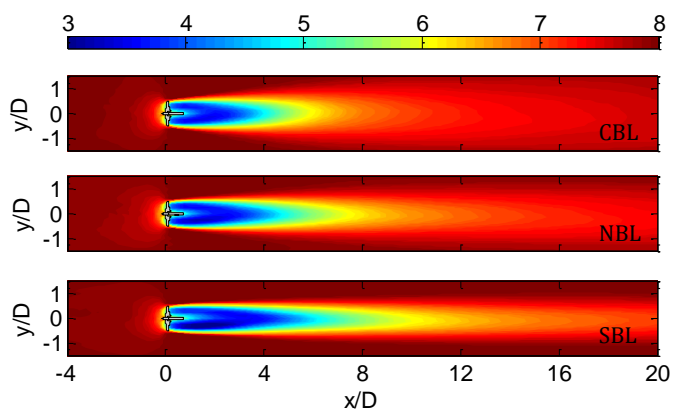

FIG. 4. Contours of the time-averaged streamwise velocity $U\left(m s^{-1}\right)$ in the horizontal $x-y$ plane at the turbine hub height for different stability conditions.

and $U_{\text {inflow }}$ is the time-averaged streamwise inflow velocity shown in Figure 2a. Again we can see that the magnitude of the velocity deficit increases with increasing the atmospheric stability for all distances downstream of the turbine. Magnusson and Smedman ${ }^{7}$ reported similar trends using the field measurements downwind of a wind turbine in the Alsvik wind farm during different atmospheric stability conditions.

Besides the velocity deficit, the other critical aerodynamic factor in wind-turbine wakes is the enhancement of turbulence intensity that is highly related to failure of wind-turbine components $^{39}$. As shown before, turbulence in the ABL is non-homogeneous and turbulence intensity in only one direction may not be enough to describe it. As a result, in order to consider the turbulence levels in all three directions, we define total turbulence intensity as $T I=\sqrt{\frac{1}{3}\left(I_{u}^{2}+I_{v}^{2}+I_{w}^{2}\right)}$, where $I_{u}, I_{v}$ and $I_{w}$ are the streamwise, spanwise and vertical turbulence intensity, respectively. Figures 6 and 7 show contours of the total turbulence intensity in a vertical $x-z$ plane through the center of the turbines as well as in a horizontal $x-y$ plane at the turbine hub height, respectively. To obtain a more quantitative understanding about the turbine-induced turbulence, both spanwise and vertical profiles of the turbulence intensity through the center of the wakes are also shown in Figure 8. As expected, the turbulence intensity has a peak at top-tip level in the middle vertical plane perpendicular to the turbines and exhibits a dual-peak pattern with larger values near the two side-tip positions at the hub-height level, which is related to the intense production of turbulent kinetic energy associated with the strong shear at those locations. It is also evident that the location and the magnitude of the maximum turbulence intensity is clearly affected by the stability condition. In particular, the higher turbulence level in the incoming flow leads to also a larger maximum wake turbulence level for the convective condition, compared with the neutral and stable ones. This is consistent with the faster recovery of the wake observed in the convective condition. In addition, as can be seen in these figures, 

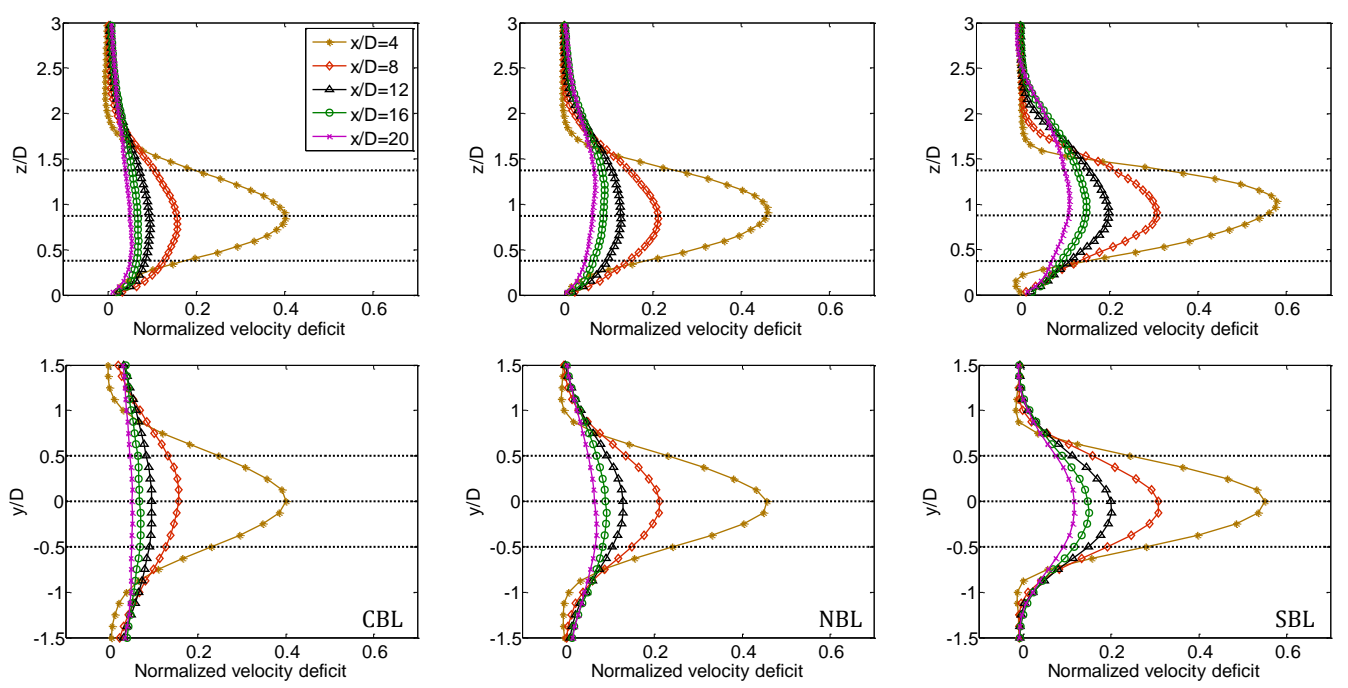

FIG. 5. Vertical (top) and spanwise (down) profiles of the normalized velocity deficit $\Delta U / U_{\text {hub }}$ through the hub level of stand-alone turbines for the unstable (left), neutral (middle), and stable (right) conditions.

the magnitude of the turbulence intensity decreases with respect to the downwind distance. However, under the stable condition, the recovery of the turbulence intensity is much slower compared with the neutral and convective cases which is mainly related to the lower turbulence intensity of the incoming wind in the stable condition compared with the neutral and convective ones.

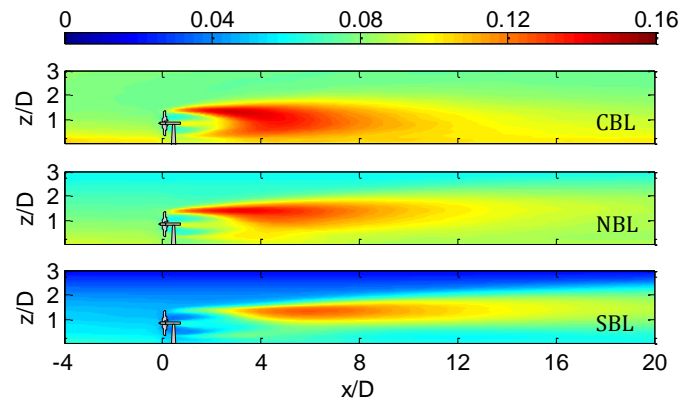

FIG. 6. Contours of the turbulence intensity $T I$ in the middle vertical $x-z$ plane perpendicular to the turbines for different stability conditions.

Figures 9 and 10 display contour plots of the vertical momentum flux $\overline{u^{\prime} w^{\prime}}$ in a vertical $x-z$ plane through the center of the turbines as well as lateral momentum flux $\overline{u^{\prime} v^{\prime}}$ in the horizontal $x-y$ plane at turbine hub height, respectively. These terms are responsible for the vertical and lateral mean kinetic energy entrainment into the wake. As can be seen in Figure 9, the turbulent momentum flux has negative values around the upper edge of the wake and positive values around the lower edge. Similar behaviour is observed in Figure 10 for the lateral momentum flux whose values are negative around one side and are positive around the other side. This can be explained by the strong entrainment of the surrounding boundary-layer flow into the wake. It is also evident that the magnitude of the momentum flux is higher in the unstable condition compared with the neutral and stable ones, which leads to a higher mean kinetic energy entrainment into the wake. The higher mean kinetic energy entrainment into the wake in the convective condition is consistent 


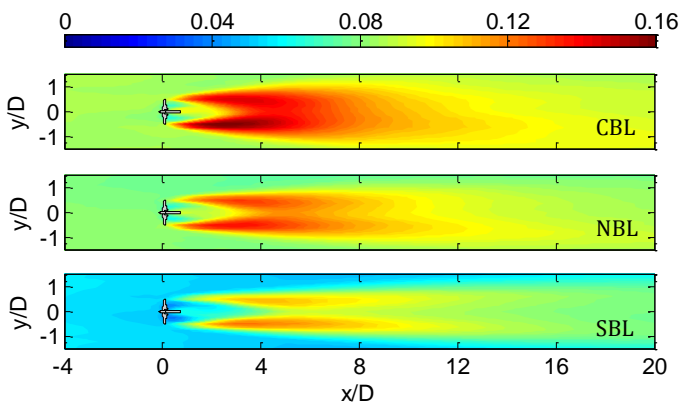

FIG. 7. Contours of the turbulence intensity $T I$ in the horizontal $x-y$ plane at the turbine hub height for different stability conditions.
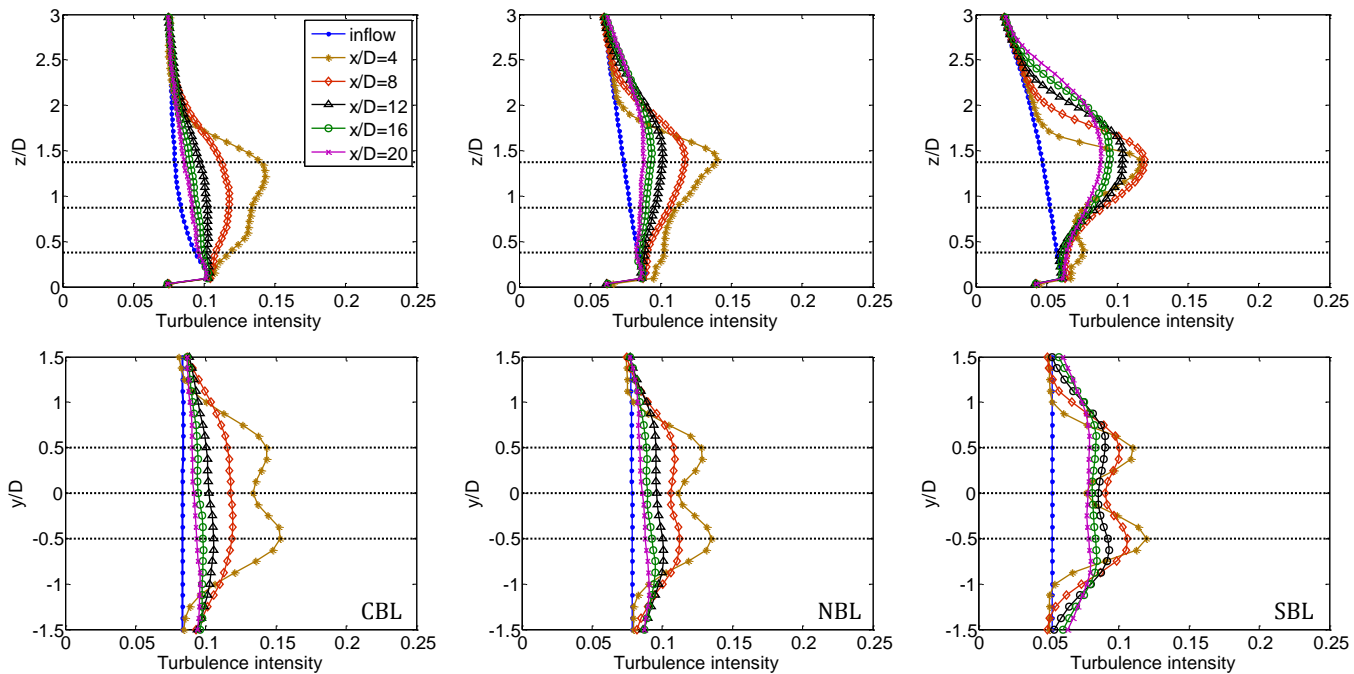

FIG. 8. Vertical (top) and spanwise (down) profiles of the turbulence intensity TI through the hub level of stand-alone turbines for the unstable (left), neutral (middle), and stable (right) conditions.

with the above-described faster wake recovery in that condition.

In validations of the same LES framework against wind-tunnel measurements, Wu and Porté-Agel ${ }^{15,40}$ showed that our LES framework yields velocity deficit and turbulence intensity results that have little resolution dependence as long as at least 7 grid points are used to cover the rotor diameter in the vertical direction, and 5 points in the spanwise direction. In the present work, the grid resolution is chosen such that 16 points in the vertical direction and 10 points in the spanwise direction cover the turbine rotor diameter. However, in order to further assess the sensitivity of the simulation results to the grid resolution, we compare the results obtained from two different spatial resolutions (see Table I). In the new simulations a similar cell aspect ratios have been used $(\Delta x: \Delta y: \Delta z \approx 3.2: 2: 1)$. Figures 11 and 12 show the profiles of the normalized velocity deficit and the turbulence intensity obtained from the two different grid resolutions. As can be seen in these figures, there is little grid resolution dependence in the profiles of velocity deficit and turbulence intensity, which indicates that the grid resolution used in the present study is fine enough to study the influence of atmospheric thermal stability on wind turbine wakes. 

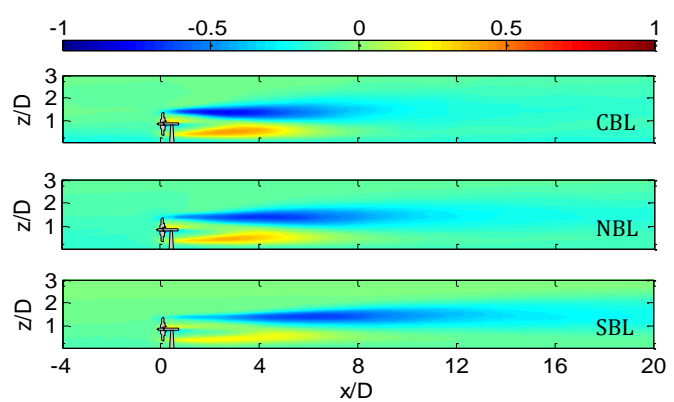

FIG. 9. Contours of the vertical momentum flux $\overline{u^{\prime} w^{\prime}}\left(m^{2} s^{-2}\right)$ in the middle vertical $x-z$ plane perpendicular to the turbines for different stability conditions.
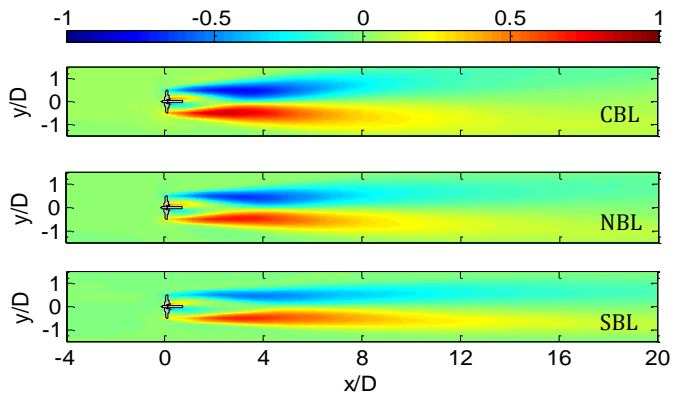

FIG. 10. Contours of the lateral momentum flux $\overline{u^{\prime} v^{\prime}}\left(m^{2} s^{-2}\right)$ in the horizontal $x-y$ plane at the turbine hub height for different stability conditions.

\section{B. Analytical wake modeling}

As shown in Figures 3 and 4, the velocity deficit in the wake has an approximately Gaussian shape after some downwind distances for all the stability conditions. This behavior was reported in previous numerical and experimental studies by Wu and Porté-Agel ${ }^{40}$, Rados et al. ${ }^{41}$, Chamorro and Porté-Agel ${ }^{42}$, and Zhang et al. ${ }^{10}$. Recently, Bastankhah and Porté-Agel ${ }^{17}$ developed a new analytical model to predict the wind velocity distribution downwind of a wind turbine by applying conservation of mass and momentum and assuming a self-similar Gaussian distribution for the velocity deficit in the wake. They tested the proposed model against high resolution wind-tunnel measurements and LES data of miniature wind turbine wakes, as well as LES data of real-scale wind-turbine wakes under neutrally-stratified conditions. In this section, we aim to extend the above-mentioned analytical model to the other atmospheric thermal stability conditions using the LES data presented in this work.

In the analytical model proposed by Bastankhah and Porté-Age ${ }^{17}$, the velocity deficit downwind of the turbine is computed as:

$$
\frac{\Delta U}{U_{\infty}}=\left(1-\sqrt{1-\frac{C_{T}}{8(\sigma / D)^{2}}}\right) \times \exp \left(-\frac{1}{2(\sigma / D)^{2}}\left\{\left(\frac{z-z_{h}}{D}\right)^{2}+\left(\frac{y}{D}\right)^{2}\right\}\right)
$$

where $C_{T}$ is the thrust coefficient of the turbine and $\sigma$ is the standard deviation of the axisymmetric Gaussian-like velocity deficit profiles at each downwind distance $x$. It should be mentioned that in this model, we only need to have a reasonable estimation of the expansion of the wake downwind of the turbine (i.e., $\sigma / D$ ).

As mentioned before, the assumption of self-similarity of the velocity deficit is critical in the above-mentioned analytical model. In order to assess the self-similar behaviour 

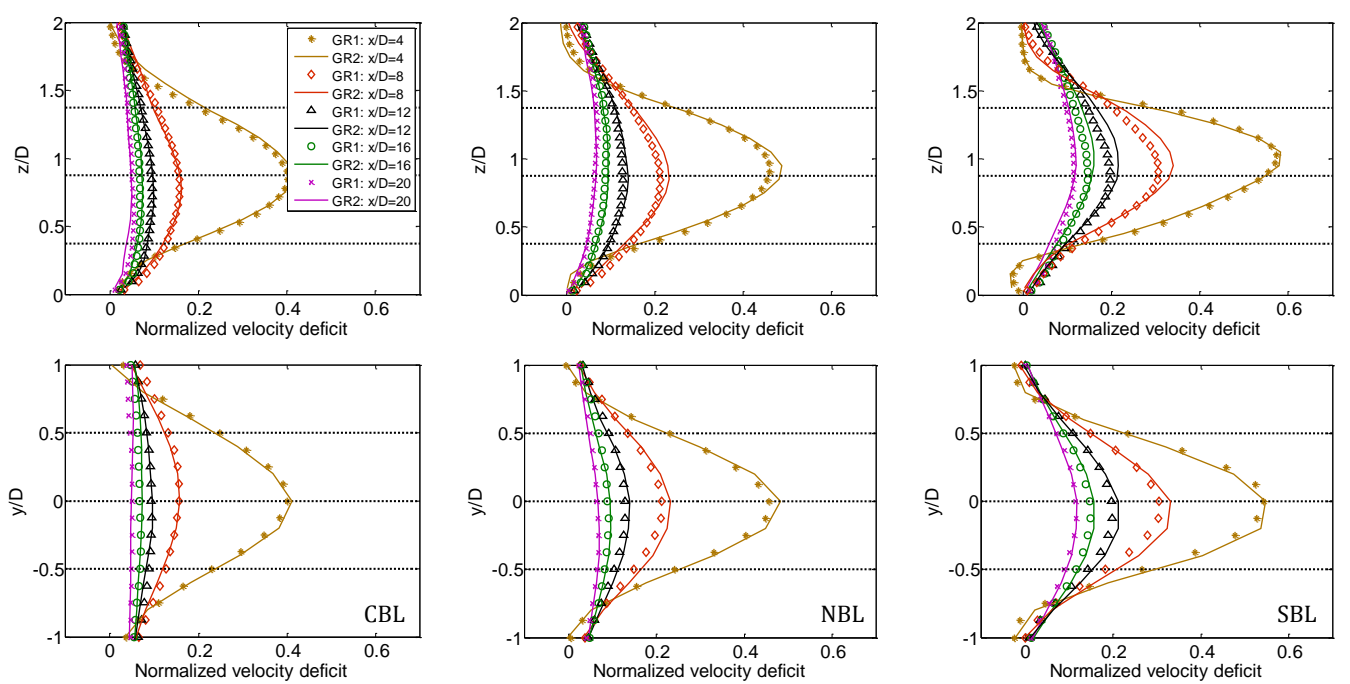

FIG. 11. Vertical (top) and spanwise (down) profiles of the normalized velocity deficit $\Delta U / U_{\text {hub }}$ through the hub height of the turbine for the unstable (left), neutral (middle), and stable (right) conditions obtained from two different grid resolutions.
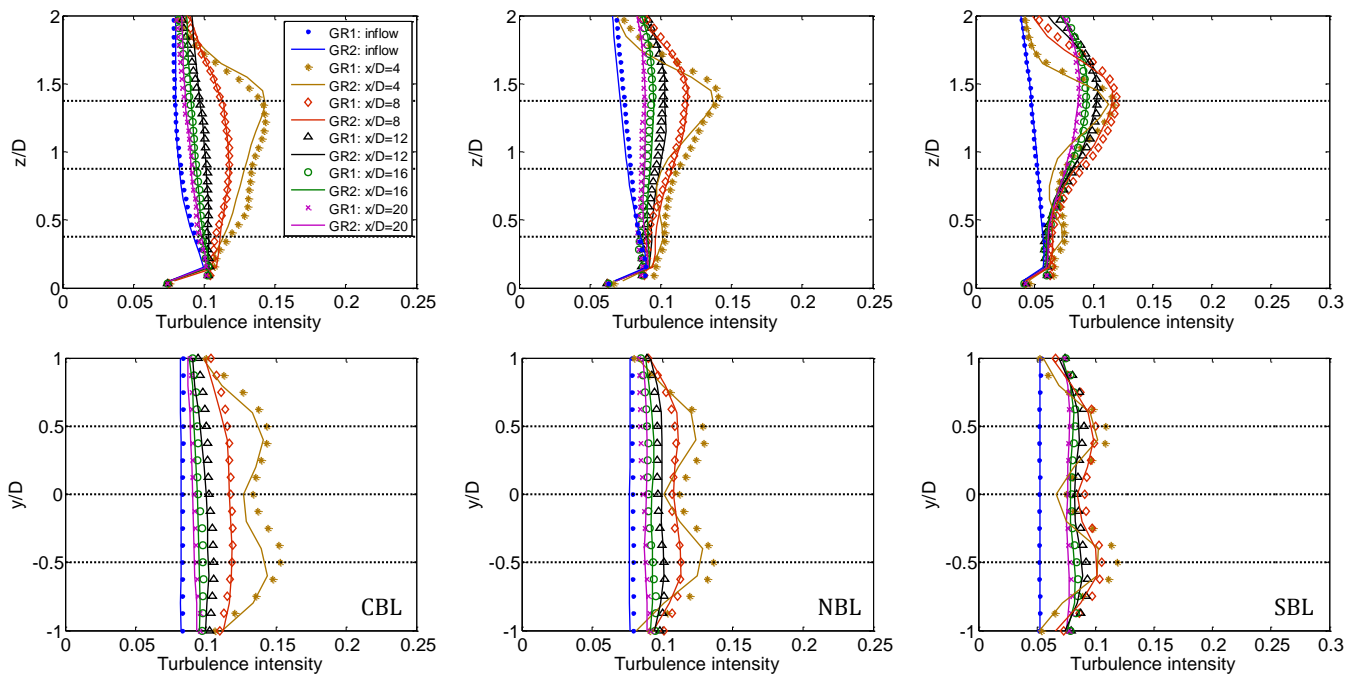

FIG. 12. Vertical (top) and spanwise (down) profiles of the turbulence intensity TI through the hub height of the turbine for the unstable (left), neutral (middle), and stable (right) conditions obtained from two different grid resolutions.

of the wake under different stability regimes, in Figure 13 the normalized velocity deficit $\left(f=\Delta U / \Delta U_{\max }\right)$ obtained at different downwind distances $(x)$ is plotted against the normalized radial distance from the center of the wake $\left(r / r_{1 / 2}\right)$. Here, the wake's halfwidth $r_{1 / 2}(x)$, defined at each $x$ as $\frac{\Delta U\left(r=r_{1 / 2}\right)}{U_{h u b b}}=\frac{1}{2} \frac{\Delta U_{\max }}{U_{h u b}}$, is used as the characteristic wake width. The figure shows that the normalized velocity-deficit profiles approximately collapse into a single Gaussian curve except at the edges of the wake. This confirms the validity of the assumption of self-similar Gaussian shape of the velocity deficit after some downwind distance $(x / D \gtrsim 4)$ for all the stability cases.

In Figure 14a, the normalized standard deviation of the Gaussian curves fitted to the vertical $\left(\sigma_{z} / D\right)$ and lateral $\left(\sigma_{y} / D\right)$ velocity deficit profiles in the wakes is plotted as a 


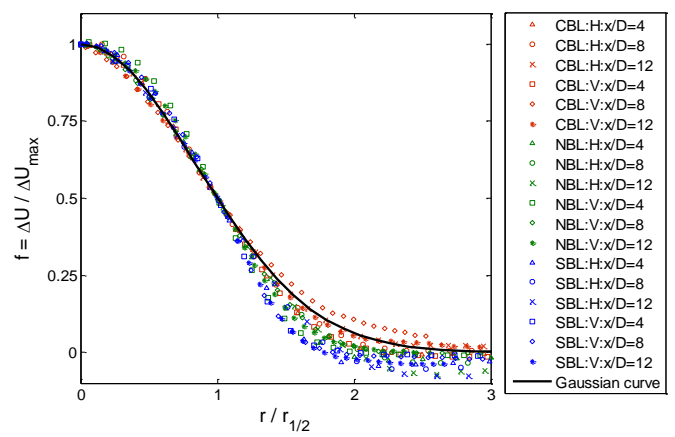

FIG. 13. Self-similar velocity-deficit profiles of the the LES data at different downwind distances. "H" and "V" represent the horizontal and vertical profiles, respectively.

function of normalized downwind distance $(x / D)$ for different cases. This figure shows that the wake expands approximately linearly in the range of $4<x / D<12$ for all the stability conditions. It is also clear that the wake recovers faster under the convective condition, in both vertical and lateral directions, compared with the neutral and stable ones. In particular, the growth rate of the wake is more than 2 times larger for the convective case than for the stable one. This is due to the fact that the higher incoming turbulence enhances the mixing processes, which results in a faster wake recovery. It is also found that, for all the stability cases, the growth rate of the wake is larger in the lateral direction compared with the vertical one. This is mainly related to the fact that the ambient turbulence of the incoming wind is stronger in the lateral direction than in the vertical one (see Figure 2), which contributes to a higher momentum flux and, consequently, a higher mean kinetic energy entrainment into the wake in the lateral direction compared with the vertical one (see Figures 9 and 10). In addition, as will be shown later, this is also consistent with the meandering characteristics of wake under different stability conditions. It should be noted that, in the analytical model of Equation 11, it is assumed that the velocity deficit has an approximately Gaussian axisymmetric shape, and thus, only one value for $\sigma$ is needed. However, the results presented here show that the velocity deficit does not show axisymmetric behavior specially under the convective condition. Hence, we extend the above-mentioned analytical model by assuming an elliptical (non-axisymmetric) Gaussian distribution for the wake. Using a two-dimensional elliptical (instead of axisymmetric) Gaussian distribution allows to account for the different lateral and vertical growth rates of the wake. Using this assumption, the velocity deficit downwind of the turbine can be computed as:

$$
\frac{\Delta U}{U_{\infty}}=\left(1-\sqrt{1-\frac{C_{T}}{8(\sigma / D)^{2}}}\right) \times \exp \left(-\frac{1}{2(\sigma / D)^{2}}\left\{\left(\frac{\sigma_{y}}{\sigma} \frac{z-z_{h}}{D}\right)^{2}+\left(\frac{\sigma_{z}}{\sigma} \frac{y}{D}\right)^{2}\right\}\right)
$$

where $\sigma=\sqrt{\sigma_{y} \sigma_{z}}$. Figure 14b shows the the variation of $\sigma / D$ as a function of normalized downwind distance.

Figure 15 shows the variation of the normalized velocity deficit, at turbine hub height, as a function of normalized downwind distance for the extended analytical model and the LES data. The prediction of the original model proposed by Bastankhah and Porté-Agel ${ }^{17}$ (Equation 11) using the growth rate of the wake only in one direction (lateral or vertical) is also shown. As seen in this figure, the extended analytical model is able to predict reasonably well the velocity deficit at hub height in the wake under different stability regimes. In addition, it is observed that considering the growth rate of the wake only in one direction, either lateral or vertical, leads to underestimation or overestimation of the velocity deficit at hub height, respectively. From this figure, although the differences are quite small in the neutral and stable cases, the difference is more noticeable in the convective one. This 

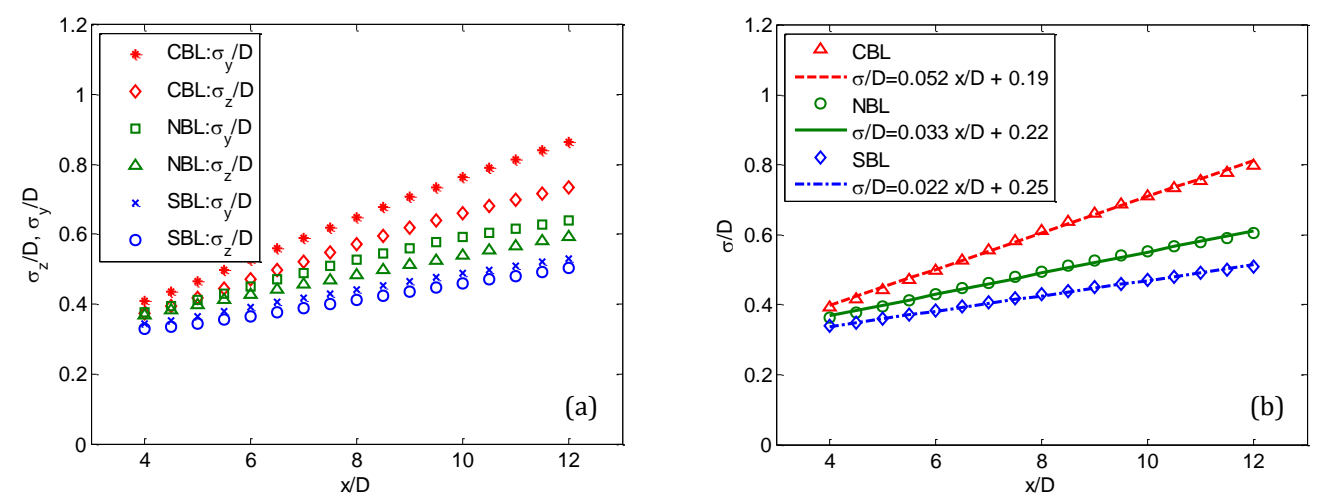

FIG. 14. Normalized standard deviation of the velocity deficit profiles under different stability conditions. a) $\sigma_{z} / D$ and $\sigma_{y} / D$, b) $\sigma / D=\sqrt{\sigma_{y} \sigma_{z}} / D$.

is consistent with the results shown in Figure 14a, where the difference between the lateral and vertical growth rates of the wake is also larger under the convective condition.
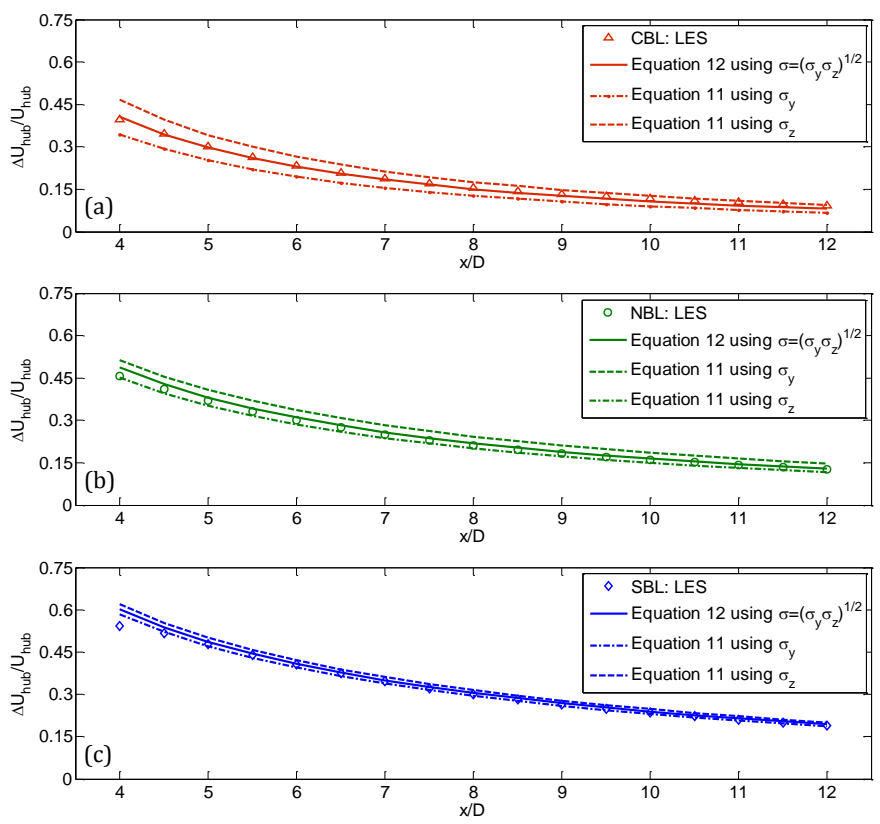

FIG. 15. Comparison of the original and extended version of Bastankhah and Porté-Agel's model for the normalized velocity deficit at hub height under a) unstable, b) neutral, and c) stable conditions.

As mentioned before and also shown in Figure 14a, the growth rate of the wake is different in different directions. In order to better understand the relation between the nonaxisymmetric growth of the wake and the wake dynamics, next, we explore the unsteady behaviour of the wake commonly regarded to "wake meandering" ${ }^{43-45}$ under different stability conditions. The meandering can be considered as a series of wake segments in which the wake center has an offset in the lateral and vertical directions ${ }^{12}$. Figure 16 shows the normalized standard deviation of the instantaneous wake center in the lateral and vertical directions as a function of downstream position. To estimate the center of the wake instantaneously, the following procedure is applied: For each time step and downwind plane, 
a multivariate Gaussian function is fitted to the instantaneous field of the velocity deficit (see Figure 17). A similar procedure was used by Keck et al. ${ }^{12}$ to quantify wake meandering by fitting a one-dimensional Gaussian function on the lateral and vertical profiles of the instantaneous velocity deficit through the hub level. As seen in Figure 16, the wake meandering in both lateral and vertical directions increases by decreasing the atmospheric stability. In addition, for all atmospheric stabilities, the wake meandering is weaker in the vertical direction than in the lateral one. This is mainly related to the fact that the presence of the ground combined with the vertical gradient of the velocity in the ABL limit the wake meandering in the vertical direction. This trend is consistent with the one reported by Keck et al. ${ }^{12}$ using the dynamic wake meandering model and the results in Figure 14a that show the growth rate of the wake is larger in the lateral direction compared with the vertical one.

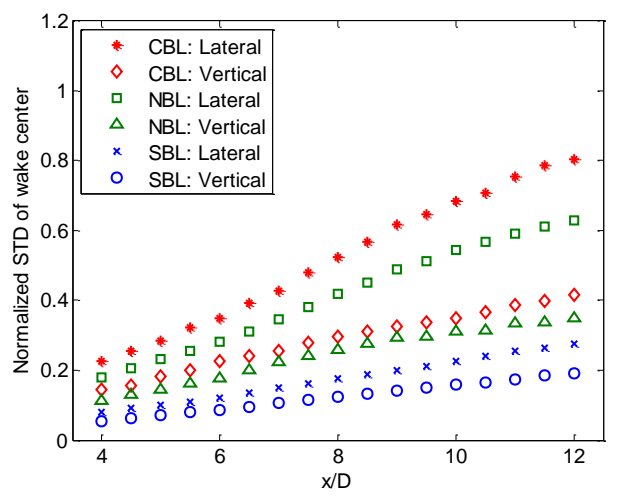

FIG. 16. Standard deviation of the wake center normalized with the rotor diameter, $D$, in the lateral and vertical directions under different thermal stratification.
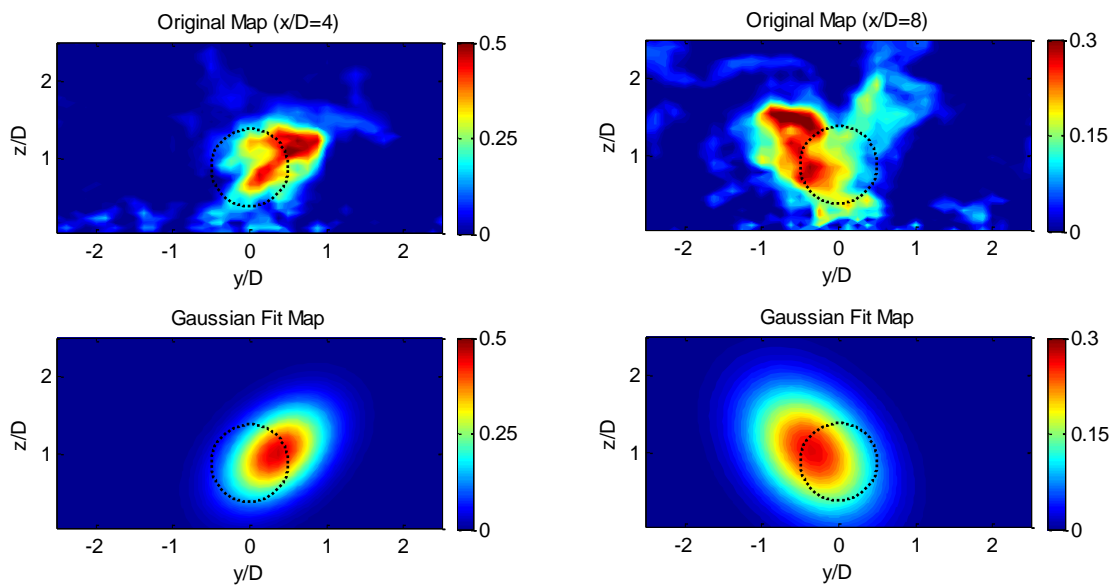

FIG. 17. Contours of the instantaneous normalized velocity deficit at $x / D=4$ (left) and $x / D=8$ (right) under the neutrally-stratified condition: top: original map, down: multivariate Gaussian fit. Dashed circle shows the location of the turbine.

\section{Resolved-scale turbulent kinetic energy (TKE) budgets}

Here, the LES results are used to investigate all the terms in the budget of the resolvedscale turbulent kinetic energy (TKE). The TKE budget analysis can provide valuable in- 
sights to better understand the energy exchanges between the ABL flow and wind-turbine wakes.

The resolved-scale TKE budget equation can be written as:

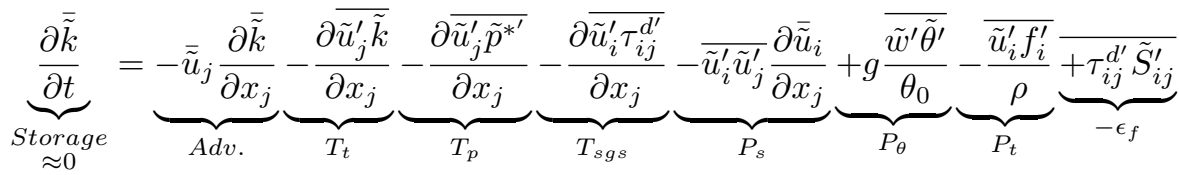

where $\overline{\tilde{k}}=0.5 \overline{\tilde{u}_{i}^{\prime} \tilde{u}_{i}^{\prime}}$ is the resolved-scale turbulent kinetic energy, the overbar $\left(^{-}\right)$denotes temporal averaging and $\tilde{u}_{i}^{\prime}=\tilde{u}_{i}-\overline{\tilde{u}}_{i}$ is the resolved velocity fluctuation. $T_{t}, T_{p}$ and $T_{s g s}$ represent the transport of the resolved-scale TKE by the fluctuations of velocity, pressure, and SGS stresses, respectively, and the sum of them is called the turbulent transport term and defined as: $T T=T_{t}+T_{p}+T_{s g s}{ }^{46} . P_{s}$ is the shear production representing the conversion of mean kinetic energy to resolved-scale TKE, $P_{\theta}$ is the buoyancy production or destruction depending on the sign of the vertical heat flux, $P_{t}$ is the rate of the work against the turbineinduced forces, and $\epsilon_{f}$ is the SGS dissipation rate which represents the energy transfer of resolved-scale TKE to the subgrid scales.

Figures 18 and 19 show contours of the turbulent kinetic energy (TKE) and the mean advection term, respectively, in a vertical $x-z$ plane through the center of the turbines. As expected, the magnitude of the TKE is higher under the convective condition compared with the neutral and stable ones. As seen in Figure 18, at top-tip region downwind of the turbine, the TKE starts to increase, reaches a maximum and then starts to decrease with downwind distance. Consistent with the previous result, Figure 19 shows that downwind of the turbine, particularly at the top-tip level, the mean advection term is negative due to the positive streamwise gradient of TKE, and it becomes positive after the TKE has reached a maximum and starts to decrease with downwind distance in the far wake. The location of the maximum TKE has been shown in these figures. These results indicate that the atmospheric stability significantly affects the location of the maximum TKE downwind of the turbine. In particular, it is further downwind under the stable condition compared with the neutral and unstable cases.

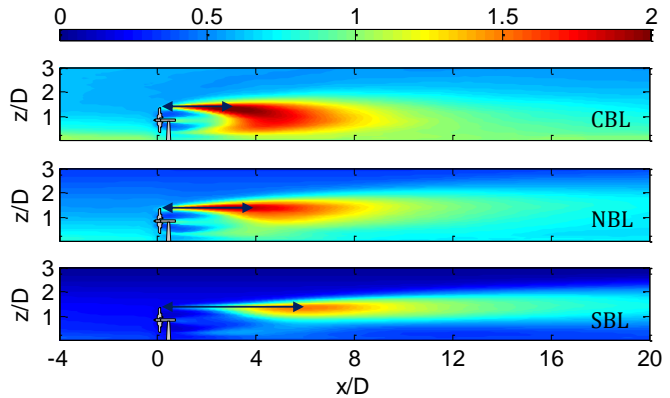

FIG. 18. Contours of the turbulent kinetic energy $\overline{\tilde{k}}\left(m^{2} s^{-2}\right)$ in the middle vertical $x-z$ plane perpendicular to the turbines for different stability conditions.

Two-dimensional contours of the turbulent transport in the vertical $x-z$ plane are shown in Figure 20. As seen in this figure, turbulent transport plays an important role in redistributing the shear-produced TKE away from the shear layer both inward and outward. Consistent with the previous results, the magnitude of the turbulent transport is larger for the unstable condition compared with the neutral and stable cases.

Figures 21 and 22 display the two-dimensional contours of the turbulence production and SGS dissipation rate (energy which is transferred to the subgrid scales) for different stability conditions. It is clear that the magnitude of the shear production is larger at the turbinetop level. This significant turbulence enhancement is associated with the combination of 


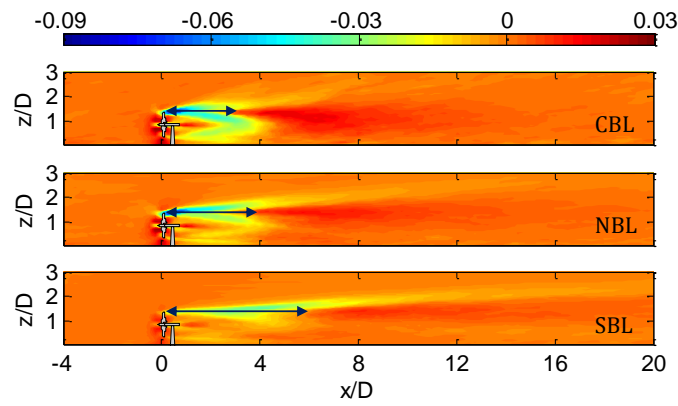

FIG. 19. Contours of the advection term $A d v .\left(m^{2} s^{-3}\right)$ in the middle vertical $x-z$ plane perpendicular to the turbines for different stability conditions.

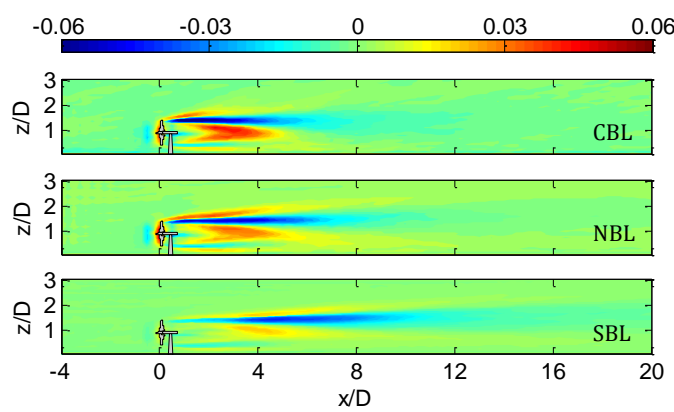

FIG. 20. Contours of the turbulent transport term $T T\left(m^{2} s^{-3}\right)$ in the middle vertical $x-z$ plane perpendicular to the turbines for different stability conditions.

a strong turbulent shear stress and a large wind shear in the turbine-induced shear layer. It is also observed that, for all the cases, the magnitude of the dissipation rate is higher at the top-tip level where the shear production is also largest. It is also found that the turbulence production and SGS dissipation rates have a peak at the turbine-top level, which is consistent with the fact that the peak shear stress also happens at this level (Figure 9).

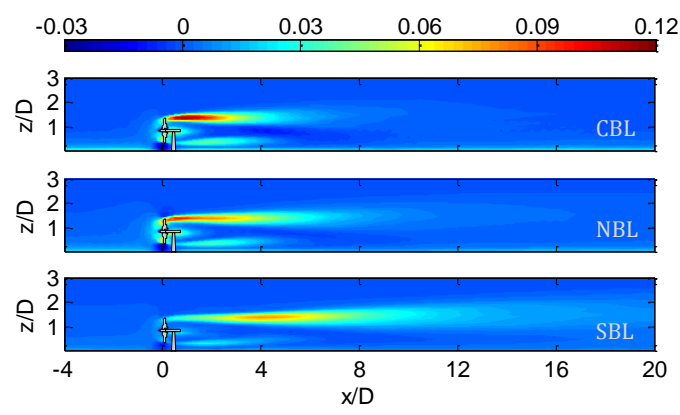

FIG. 21. Contours of the TKE production $P_{\text {total }}=P_{s}+P_{\theta}\left(m^{2} s^{-3}\right)$ in the middle vertical $x-z$ plane perpendicular to the turbines for different stability conditions.

\section{SUMMARY}

In this study, large-eddy simulation (LES), coupled with a turbine model, was used to investigate the effect of atmospheric stability on wind-turbine wakes. In the simulations, the 


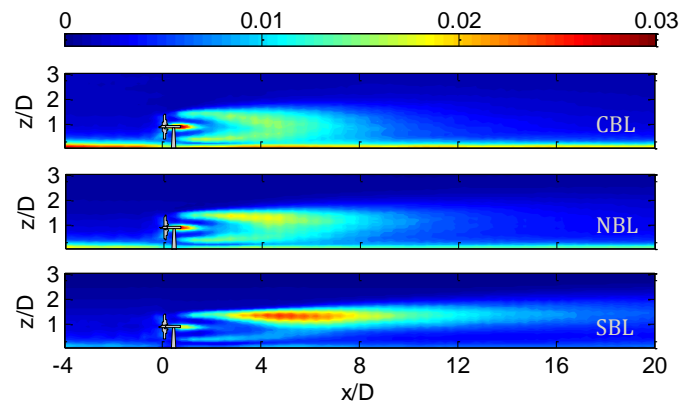

FIG. 22. Contours of the SGS dissipation rate $\epsilon_{f}\left(m^{2} s^{-3}\right)$ in the middle vertical $x-z$ plane perpendicular to the turbines for different stability conditions.

tuning-free Lagrangian scale-dependent dynamic models were used to model the subgridscale turbulent fluxes, and the turbine-induced forces (e.g. lift and drag) were parameterized with the actuator disk model with rotation.

The simulation results show that atmospheric thermal stability has a significant influence on the spatial distribution of the mean velocity deficit, turbulence intensity, and turbulent momentum fluxes in the turbine wakes. In particular, the wake recovers faster under the convective condition compared with the neutral and stable cases. This enhancement in the wake recovery rate is related to the higher turbulence level of the incoming wind in the unstable condition, which leads to a higher turbulent entrainment flux into the wake. For the particular cases considered in this study, the growth rate of the wake is more than 2 times larger for the convective case than for the stable one. It is also shown that atmospheric stability has a significant effect on the meandering characteristics of the wake. Specifically, for a given distance downwind of the turbine, wake meandering is stronger under the convective condition compared with the neutral and stable ones. In addition, for all the stability cases considered in this study, the growth rate of the wake and wake meandering are smaller in the vertical direction compared with the lateral one. This is mainly related to the the presence of the ground combined with the different turbulence levels of the incoming wind in different directions. The results indicate that the streamwise turbulence intensity alone is not sufficient to describe the impact of the atmospheric stability on wind-turbine wakes, and turbulence intensities in all three directions must be considered.

Inspired by the obtained results, a generalized version of the recently developed analytical wake model by Bastankhah and Porté-Agel ${ }^{17}$ is proposed which is able to account for the different lateral and vertical growth rates of the wake. The new analytical model is based on applying conservation of mass and momentum and assuming an elliptical (instead of axisymmetric) Gaussian distribution for the velocity deficit in the wake. The LES results confirm that the velocity deficit profile has a nearly self-similar Gaussian shape after some downwind distance under different stability conditions. In addition, the results show that the new analytical model is able to accurately predict the velocity deficit in the wake of a stand-alone wind turbine.

Finally, the LES data were used to analyze the resolved-scaled TKE budget inside the wake. The results reveals that atmospheric stability significantly affects the magnitude and spatial distribution of the turbulence production, dissipation and transport terms. It is found that, for all the cases, the turbulence production and dissipation reach a peak around the upper edge of the wake, due to the higher wind shear and turbulent fluxes in that region. Also, the magnitude of the turbulence production, downwind of the turbine, decreases with increasing atmospheric stability. Moreover, it is shown that the thermal stability has a great influence on the location of the maximum TKE production and the extent of the near wake region downwind of the turbine. Particularly, it is further downwind under the stable condition compared with the neutral and unstable cases.

Future research will address the effect of thermal stratification on the structure and 
characteristics of wind-turbine wakes and their interaction with the ABL inside wind farms.

Acknowledgements. This research was supported by the Swiss National Science Foundation (grants 200021-132122 and IZERZ0-142168) and the Swiss Innovation and Technology Committee (CTI) within the context of the Swiss Competence Center for Energy Research FURIES: Future Swiss Electrical Infrastructure. Computing resources were provided by the Swiss National Supercomputing Center (CSCS) under project ID s306.

${ }^{1}$ L. J. Vermeer, J. N. Sørensen, A. Crespo, Wind turbine wake aerodynamics, Prog. Aerosp. Sci. 39 (2003) $467-510$

${ }^{2}$ J. N. Sørensen, Aerodynamic aspects of wind energy conversion, Annu. Rev. Fluid Mech. 43 (2011) $427-448$.

${ }^{3}$ R. J. Barthelmie, S. C. Pryor, S. T. Frandsen, K. S. Hansen, J. G. Schepers, K. Rados, W. Schlez, A. Neubert, L. E. Jensen, N. S., Quantifying the impact of wind turbine wakes on power output at offshore wind farms, Atmos. Ocean. Tech. 27 (8) (2010) 1302-1317.

${ }^{4}$ R. Stull, An introduction to boundary-layer meteorology, Kluwer Academic Publishers, Dordrecht, 1988.

${ }^{5}$ J. Garratt, The atmospheric boundary layer, Cambridge University Press, U.K., 1994.

${ }^{6} \mathrm{R}$. W. Baker, S. N. Walker, Wake measurements behind a large horizontal axis wind turbine generator, Solar Energy 33 (1984) 5-12.

${ }^{7}$ M. Magnusson, A. S. Smedman, Influence of atmospheric stability on wind turbine wakes, Wind Eng. 18 (1994) 139-152.

${ }^{8}$ R. J. Barthelmie, L. E. Jensen, Evaluation of wind farm efficiency and wind turbine wakes at the nysted offshore wind farm, Wind Energy 13 (2010) 573-586.

${ }^{9} \mathrm{G}$. V. Iungo, F. Porté-Agel, Volumetric lidar scanning of wind turbine wakes under convective and neutral atmospheric stability regimes, Journal of Atmospheric and Oceanic Technology 31 (10) (2014) 2035-2048.

${ }^{10}$ W. Zhang, C. D. Markfort, F. Porté-Agel, Wind-turbine wakes in a convective boundary layer: a windtunnel study, Boundary-Layer Meteorol. 146 (2013) 161-179.

${ }^{11}$ P. E. Hancock, F. Pascheke, Wind-tunnel simulation of the wake of a large wind turbine in a stable boundary layer: Part 2, the wake flow, Boundary-Layer Meteorol. 151.1 (2014) 23-37.

${ }^{12}$ R. E. Keck, M. Mare, J. M. Churchfield, S. Lee, G. Larsen, On atmospheric stability in the dynamic wake meandering model, Wind Energy 17 (2014) 1689-1710.

${ }^{13}$ M. J. Churchfield, S. Lee, J. Michalakes, P. J. Moriarty, A numerical study of the effects of atmospheric and wake turbine dynamics, J. Turbu. 13 (2012) N14.

${ }^{14}$ R. Stoll, F. Porté-Agel, Dynamic subgrid-scale models for momentum and scalar fluxes in large-eddy simulations of neutrally stratified atmospheric boundary layers over heterogeneous terrain, Water Resour. Res. 42.1 (2006) W01409.

${ }^{15}$ Y. T. Wu, F. Porté-Agel, Large-eddy simulation of wind-turbine wakes: evaluation of turbine parametrisations, Boundary-Layer Meteorol. 138 (3) (2011) 345-366.

${ }^{16}$ F. Porté-Agel, Y. T. Wu, H. Lu, R. J. Conzemius, Large-eddy simulation of atmospheric boundary layer flow through wind turbines and wind farms, J. Wind Eng. Ind. Aerodyn. 99 (4) (2011) 154-168.

${ }^{17}$ M. Bastankhah, F. Porté-Agel, A new analytical model for wind-turbine wakes, Renewable Energy 70 (2014) 116-123.

18 J. Smagorinsky, General circulation experiments with the primitive equations, Mon. Weather Rev. 91 (3) (1963) 99-164.

${ }^{19}$ M. Germano, U. Piomelli, P. Moin, W. Cabot, A dynamic subgrid-scale eddy viscosity model, Phys. Fluids A 3 (1991) 1760-1765.

${ }^{20}$ P. Moin, K. D. Squires, S. Lee, A dynamic subgrid-scale model for compressible turbulence and scalar transport, Phys. Fluids A: Fluid Dynamics 3 (1991) 2746.

${ }^{21}$ F. Porté-Agel, C. Meneveau, M. B. Parlange, A scale-dependent dynamic model for large-eddy simulation: application to a neutral atmospheric boundary layer, J. Fluid Mech. 415 (2000) 261-284.

${ }^{22} \mathrm{~F}$. Porté-Agel, A scale-dependent dynamic model for scalar transport in large-eddy simulations of the atmospheric boundary layer, Boundary-Layer Meteorol. 112 (2004) 81-105.

${ }^{23}$ Y.-T. Wu, F. Porté-Agel, Modeling turbine wakes and power losses within a wind farm using LES: An application to the Horns Rev offshore wind farm, Renewable Energy 75 (2015) 945-955.

${ }^{24}$ J. D. Albertson, M. B. Parlange, Surfaces length scales and shear stress: implications for land-atmosphere interactions over complex terrain, Water Resour. Res. 35 (1999) 2121-2132.

${ }^{25} \mathrm{~F}$. Porté-Agel, Y. T. Wu, C. H. Chen, A numerical study of the effects of wind direction on turbine wakes and power losses in a large wind farm, Energies 6(10) (2013) 5297-5313.

${ }^{26}$ S. Zilitinkevich, I. Esau, A. Baklanov, Further comments on the equilibrium height of neutral and stable planetary boundary layers, Q. J. R. Meteorol. Soc. 133 (2007) 265-271.

${ }^{27}$ A. Peña, S. E. Gryning, C. B. Hasager, Comparison mixing-length models of the diabatic wind profile over homogeneous terrain, Theor. Appl. Climatol. 100 (2010) 325-335.

${ }^{28}$ Y. H. Tseng, C. Meneveau, M. B. Parlange, Modeling flow around bluff bodies and predicting urban dispersion using large eddy simulation, Environ. Sci. Technol. 40 (2006) 2653-2662.

${ }^{29}$ M. Abkar, F. Porté-Agel, A new boundary condition for large-eddy simulation of boundary-layer flow over surface roughness transitions, J. Turbul. 13 (23) (2012) 1-18. 
${ }^{30}$ S. A. Orszag, Transform method for calculation of vector coupled sums: Application to the spectral form of the vorticity equation, J. Atmos. Sci. 27 (1970) 890-895.

${ }^{31}$ C. Canuto, M. Hussaini, A. Quarteroni, T. Zang, Spectral Methods in Fluid Dynamics, Springer, 1988.

${ }^{32}$ A. Monin, M. Obukhov, Basic laws of turbulent mixing in the ground layer of the atmosphere, Tr. Akad. Nauk SSSR Geophiz. Inst. 24 (1954) 163-187.

${ }^{33} \mathrm{C}$. Moeng, A large-eddy simulation model for the study of planetary boundary-layer turbulence, J. Atmos. Sci. 46 (1984) 2052-2062.

${ }^{34}$ R. Stoll, F. Porté-Agel, Surface heterogeneity effects on regional-scale fluxes in stable boundary layers: surface temperature transitions, J. Atmos. Sci. 66(2) (2009) 412-431.

${ }^{35}$ J. A. Businger, J. C. Wyngaard, Y. Izumi, E. F. Bradley, Flux-profile relationships in the atmospheric surface layer, J. Atmos. Sci. 28 (1971) 181-189.

${ }^{36}$ K. S. Hansen, R. J. Barthelmie, L. E. Jensen, A. Sommer, The impact of turbulence intensity and atmospheric stability on power deficits due to wind turbine wakes at horns rev wind farm, Wind Energy 15 (2012) 183-196.

${ }^{37}$ M. Magnusson, A. S. Smedman, Air flow behind wind turbines, J. Wind Eng. Ind. Aerodyn. 80(1) (1999) 169-189.

${ }^{38}$ R. J. Barthelmie, S. T. Frandsen, M. N. Nielsen, S. C. Pryor, P. E. Rethore, H. E. Jørgensen, Modelling and measurements of power losses and turbulence intensity in wind turbine wakes at middelgrunden offshore wind farm, Wind Energy 10(6) (2007) 517-528.

${ }^{39}$ A. Rosen, Y. Sheinman, The power fluctuations of a wind turbine, J. Wind Eng. Ind. Aerodyn. 59 (1996) $51-68$.

${ }^{40}$ Y. T. Wu, F. Porté-Agel, Atmospheric turbulence effects on wind-turbine wakes: an LES study, Energies 5 (12) (2012) 5340-5362.

${ }^{41}$ K. Rados, G. Larsen, R. Barthelmie, W. Schlez, B. Lange, G. Schepers, T. Hegberg, M. Magnisson, Comparison of wake models with data for offshore wind farms, Wind Eng. 25 (2001) 271-280.

${ }^{42}$ L. Chamorro, F. Porté-Agel, A wind-tunnel investigation of wind-turbine wakes: Boundary-layer turbulence effects, Boundary-Layer Meteorol. 132 (2009) 129-149.

${ }^{43} \mathrm{~F}$. Bingol, J. Mann, C. Larsen, Light detection and ranging measurements of wake dynamics. part1: one-dimensional scanning, Wind Energy 13 (2010) 51-61.

${ }^{44}$ G. España, S. Aubrun, S. Loyer, P. Devinant, Wind tunnel study of the wake meandering downstream of a modelled wind turbine as an effect of large scale turbulent eddies, J. Wind Eng. Ind. Aerodyn. $101(0)$ (2012) 24-33.

${ }^{45}$ S. Kang, X. Yang, F. Sotiropoulos, On the onset of wake meandering for an axial flow turbine in a turbulent open channel flow, J. Fluid Mech. 744 (2014) 376-403.

${ }^{46}$ M. J. Dwyer, E. G. Patton, R. H. Shaw, Turbulent kinetic energy budgets from a large-eddy simulation of airflow above and within a forest canopy, Boundary-Layer Meteorol. 84.1 (1997) 23-43. 